\title{
Asymmetric Pentacene Derivatives for Organic Light-Emitting Diodes
}

Bo-Bin Jang, ${ }^{\dagger}$ Sang Ho Lee ${ }^{\dagger}$ and Zakya H. Kafafi*

Optical Sciences Division, U.S. Naval Research Laboratory, Washington, D.C. 20375

* Electronic mail: kafafi@nrl.navy.mil

${ }^{\dagger}$ Also at SFA Inc., Largo, MD 20774

\section{Supporting Information}

\section{Reference}

(40) Anderson, J. D.; McDonald, E. M.; Lee, P. A.; Anderson, M. L.; Ritchie, E. L.; Hall, H. K.; Hopkins, T.; Mash, E. A.; Wang, J.; Padias, A.; Thayumanavan, S.; Barlow, S.; Marder, S. R.; Jabbour, G. E.; Shaheen, S.; Kippelen, B.; Peyghambarian, N.; Wightman, R. M.; Armstrong, N. R. J. Am. Chem. Soc. 1998, 120, 9646. 